\title{
Pseudomonas aeruginosa chemotaxis strategy
}

\begin{abstract}
It would be interesting to determine if dopamine (a monoamine catecholamine compounds) in the environment affect bacterial social and motility behaviors. Understanding this interaction in $P$. aeruginosa species could provide new insights into the regulation of the virulence of this pathogen in both environmental and clinical settings. In this case report, we examined two clinical isolates of Pseudomonas aeruginosa for their ability to produce biofilm with a chemotaxis behavior for the dopamine to explain this social behavior.
\end{abstract}

Keywords: motility, p. aeruginosa, virulence, biofilm, chemotaxis, dopamine
Volume 2 Issue 2 - 2017

\author{
Meliani A,' Bensoltane A, ${ }^{2}$ Benlebna F,' \\ Bendahou $\mathrm{K}^{\prime}$ \\ 'Department of Biology, University of Mustapha Stambouli, \\ Algeria \\ ${ }^{2}$ Department of Biology, University Oran (Es-senia), Algeria
}

Correspondence: Amina Meliani, department of biology, university of Mustapha Stambouli, Mascara,Avenue Cheikh El Khaldi, Mascara 29000,Algeria, Tel 002/355/805873,

Email ameliani2003@yahoo.fr

Received: January 27, 2016 | Published: February 20, 2017
Abbreviations: CF, cystic fibrosis; UTIs, urinary and genital tract infections; CLSI, clinical and laboratory standards institute; TFP, type IV Pili; PGI, pseudomonas genital isolate

\section{Introduction}

Pseudomonas aeruginosa is one of the most important opportunistic human pathogens with the ability of biofilm formation on different abiotic surfaces, including artificial implants, endotracheal tubes, urinary catheters and contact lenses. ${ }^{1}$ It is one of the most important nosocomial pathogens which can lead to death especially among immune compromised patients ${ }^{2}$ and patients suffering from cystic fibrosis, malignancy, burns or traumatic wounds. ${ }^{3}$ Pseudomonas aeruginosa is also the major pathogen contributing to the morbidity and mortality associated with cystic fibrosis (CF), causing chronic lung infections and makes a considerable contribution to the burden of hospital-acquired infections, ${ }^{4}$ where it is a frequent cause of respiratory and urinary tract infections, a common cause of hospitalacquired pneumonia, healthcare-associated pneumonia and ventilatorassociated pneumonia. ${ }^{5}$

Thus, attempts of treatment of $P$. aeruginosa from patients through intense antimicrobial therapy may lead to significant selection of resistance strains in care units of the hospitals. ${ }^{2}$ The most motile bacteria can sense and respond to low concentrations of organic compounds in their environment by the process of chemotaxis. ${ }^{6}$ This one is considered as the first step or strategy for an inter kingdom communication. We report a case of rapidly fatal Pseudomonas aeruginosa community-acquired urinary and genital tract infections (UTIs) in a previously 59-year and 25-year woman. We discuss the chemotaxis behavior vis a vis dopamine of two $P$. aeruginosa isolates from urinary tract and genital tract.

\section{Case presentation}

59 and 25 year female patients were admitted in the surgery ward with complaints of difficulty in passing urine since one month, a several history of fever, dorsal pain, severe dyspnea and other signs of a systemic inflammatory response. There was no history of burning micturition and hematuria and no history of chronic illness suggestive of immune compromised status. The case was provisionally diagnosed as stricture of urethra with UTI. Two samples from urine and genital tract were received for culture and sensitivity and processed by routine semi quantitative method. On blood agar and Muller Hinton agar rough wrinkled yellow pigmented colonies were grown and on MacConkey agar, nonlactose-fermenting colonies were grown. Gramnegative, motile, oxidase positive, non fermenter bacilli were isolated. The isolate were further identified as $P$. aeruginosa by the API NE automated identification system (bioMérieux). Antibiotic sensitivity was done by disc diffusion method using Clinical and Laboratory Standards Institute (CLSI) guidelines. The isolates were resistant to Aztreonam, amoxicillin/clavulanate, cefazolin, amoxicillin and penicillin.

For static biofilm formation, the two clinical isolates were inoculated to $1.0 \mathrm{ml} \mathrm{LB}$ and grown with shaking at $37^{\circ} \mathrm{C}$ overnight. This culture was diluted 1:100 in LB and grown in triplicate in a polystyrene 96well plate overnight at $37^{\circ} \mathrm{C}$ without agitation, wells without bacteria served as blanks. The biofilm formation protocol was adapted from that of O'Toole GA et al. ${ }^{7}$ For motility, swimming (Tryptone swim plates $(1 \%$ tryptone, $0.5 \% \mathrm{NaCl}, 0.3 \%$ agar $)$ were inoculated. Swarm plates were composed of $0.5 \%$ Bacto Agar and $8 \mathrm{~g}$ of nutrient broth/ liter, supplemented with $5 \mathrm{~g}$ of dextrose/liter. However, for twitching motility, cells were stab inoculated with a toothpick through a thin LB agar layer ( $1 \%$ agar) to the bottom of the petri dish. The chemotactic response was quantified by a slightly modified version of the chemo attraction capillary assay vis a vis dopamine.

\section{Discussion}

Bacterial motility plays a pivotal role in microbial surface colonization and the spreading of bacteria across the surface. These motilities contribute to the formation of structured surfaceassociated communities of bacteria called biofilms. ${ }^{8}$ Biofilm infections are difficult to eradicate with antimicrobial treatment and in vitro susceptibility tests show considerable resistance of biofilm cells to killing. ${ }^{9}$ Such communities are problematic in a number of environmental and clinical settings due to their enhanced resistance to antimicrobial agents..$^{10}$ The identification of compounds that impede bacterial motility offers the potential application of such compounds for the limitation of bacterial surface colonization. For this reason, investigations on the swimming behavior of bacteria are crucial to understand chemotaxis, biofilm formation and virulence. ${ }^{11}$ 
According to most reviews, the success of $P$. aeruginosa in various environments is attributed to its broad metabolic versatility and its elaboration of many cell-associated and secreted virulence/survival factors. ${ }^{12}$ Pseudomonas aeruginosa, it can undergo the flagellummediated swimming motility and the surface-associated swarming and twitching motilities, which are predominantly mediated by hyper flagellation and type-IV Pili, respectively. ${ }^{13}$ Two surface organelles, flagella and type IV Pili (TFP), affect virulence in acute and chronic models of Pseudomonas disease. Bacteria lacking flagella caused less inflammation and death than wild-type counter parts in a murine model of acute pneumonia. ${ }^{14}$ To the best of our knowledge, there are any report describing $P$. aeruginosa chemotaxis vis a vis levodopa. Regardless, one of the levodopa characteristics as precursor of the most efficient transmitter in dopaminergic neurons from the viewpoint of therapy, it seems that $P$. aeruginosa isolates respond positively to the levodopa with a swarming and swimming motility. Swarming motility occurs when particular amino acids serve as sole nitrogen sources..$^{15}$ This motility is promoted by the production of bacterial wetting agents, such as HAAs and mono and di-rhamnolipids. ${ }^{16}$

The scientific literature revealed that the role of biofilms in many infectious diseases including urinary tract infections and chronic diseases has been demonstrated and they are thus of clinical concern. Formation of a biofilm is a particularly important aspect of bacterial pathogenesis as it enables the microbe to avoid the attack from therapeutic antimicrobials and the host's immune defences. ${ }^{17}$ Biofilm formation and motility behavior amongst the isolates also differed in the chemotaxis behavior, we observed that the clinical isolates form static biofilms and their behavior motility is distinct profiles (Figure 1). However, it is not surprising that isolates retain the ability to get a chemotaxis behavior vis a vis glucose, tryptophan (data not shown), but for levodopa further investigation should be focused on the discovery of this link. Dopamine (monoamine catecholamine) plays an important role in motor behavior and neuromodulation. As well as having endocrinological roles, dopamine, noradrenaline and adrenaline function as neurotransmitters. ${ }^{18}$ According to Freestone et al. ${ }^{19}$ clinical levels of catecholamines also stimulated $P$. aeruginosa biofilm formation on endotracheal tubing. Likewise, using in vitro growth and virulence assays and an ex vivo model of infection using human respiratory epithelium $5 \mu \mathrm{M}$ dopamine were found to markedly increase $P$. aeruginosa growth via induction of synthesis of the siderophore pyoverdine and provision of $\mathrm{Fe}$ from serum transferring. ${ }^{19}$

Interestingly, the results of the present report revealed a significant diversity in biofilm formation within the clinical isolates with OD590 readings of 0,045 and 0,013 for the urinary and genital isolates, respectively (Figure 2). The urinary isolate exhibit swarming and twitching motility. Twitching motility was distinguished by the presence of an interstitial twitch zone formed by colony expansion. The urinary isolate exhibiting twitching motility formed flat spreading colonies with a characteristic "rough" appearance and a twitching zone consisting of a very thin layer of cells observed as a halo around the colony (PUI 48h). However for the genital isolate only a swimming motility was signaled. Nonetheless, it is notable that the di-rhamnolipids were released in the medium and in the case of swimming motility (PGI 48h). Chemotaxis test "self made" was positive for glucose, tryptophan and levodopa with distinct response. The quantitative assessment of chemotaxis toward levodopa revealed that enumerated CFUs of the genital isolate (PGI) was higher $\left(29.10^{2}\right.$ CFUs. $\left.\mathrm{ML}^{-1}\right)$ than the urinary isolate $\left(13.10^{2} \mathrm{CFUs}_{\mathrm{ML}}{ }^{-1}\right)$ because of the evolved sensors for detecting eukaryotic hormones.

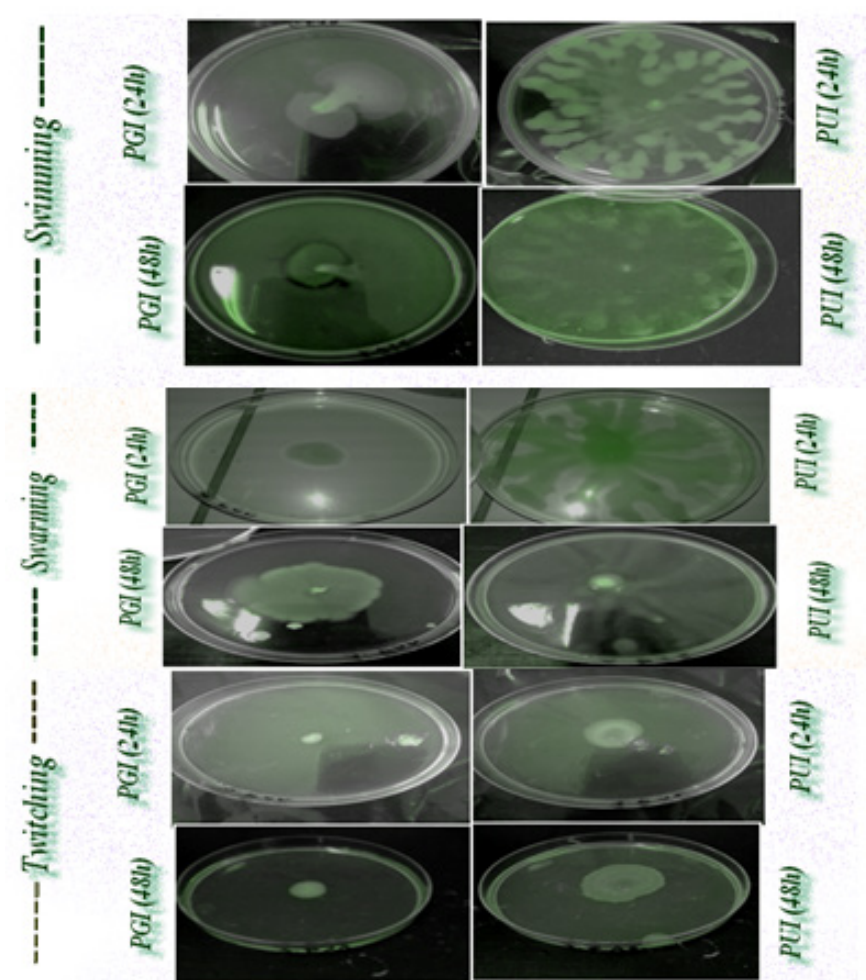

Figure I Clinical isolates display variable swarming and swimming phenotypes. The urinary isolate (PUI) represent a swarming and twitching phenotypes, however the genital isolate (PGI) displayed more a swimming phenotypes. Swimming, swarming and twitching motility is dependent on agar \% and drying time under laminar flow. Urinary isolate displays moderate to maximum tendril expression.

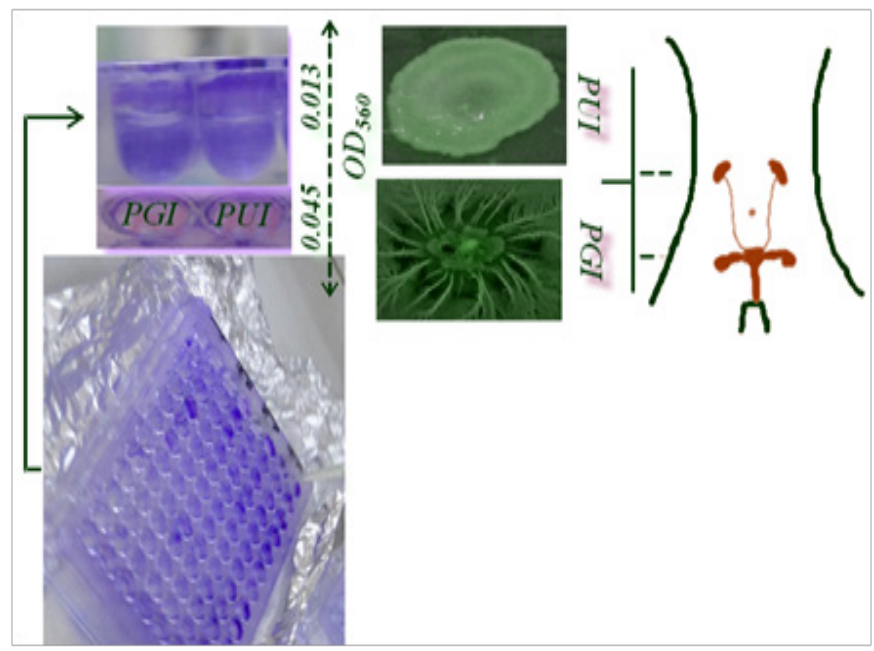

Figure 2 Microtiter plate method showing moderate biofilm production differentiated by crystal violet stain in 96-well tissue culture plate, just two clinical isolates were selected for this report the PUI (Pseudomonas urinary isolate) and PGI (Pseudomonas genital isolate).

Thus, urinary and genital tract can be colonized by $P$. aeruginosa isolates that display heterogeneity in both motility and biofilm phenotype. The results of the present report could provide a better understanding of the bacterial chemotaxis to these compounds and contribute useful information for the design of novel agents to control the phenotypes of the opportunistic pathogen P. aeruginosa. Based 
on our findings, we seek some evidence which support the hypothesis that $P$. aeruginosa can sense dopamine and respond to dopaminergic sensory neurons. Singh et al. ${ }^{20}$ reported that bacteria recognize our signalling molecules, so the obvious question that follows is whether the chemical signals of prokaryotes are able to con-vey information to eukaryotes and because of $P$. aeruginosa QS systems and the homoserine lactones of the infection stade. Furthermore, previous studies have shown that bacteria have evolved the ability to recognize catecholamine as signalling molecules in nature to find a suitable host. ${ }^{18}$

The signaling network of Pseudomonas aeruginosa is perhaps one of the most complex systems known and to date, is the best studied among all microorganism systems. It consists of multiple interconnected signaling layers that coordinately regulate virulence and persistence, driving the emergence of $P$. aeruginosa from the enormous number of species that comprise the biodiverse bacterial domain to join an elite group of a few dozen that pose a major threat to humans. ${ }^{21}$ Intriguingly, many studies were undertaken about the microbial endocrinology but how about the microbial neurology. Here, the question arises why should bacteria have evolved the ability to recognize a neurotransmitter such as dopamine with a specific motility mediated chemotaxis?

\section{Acknowledgements}

None.

\section{Conflict of interest}

The author declares no conflict of interest.

\section{References}

1. Knezevic P, Petrovic O. A colorimetric microtiter plate method for assessment of phage effect on Pseudomonas aeruginosa biofilm. J Microbiol Methods. 2008;74(2-3):114-118.

2. Tsakris A, Pournaras S, Woodford N, et al. Outbreak of infections caused by Pseudomonas aeruginosa producing VIM-1 carbapenemase in Greece. J Clin Microbiol. 2000;38(3):1290-1292.

3. Karakoç B, Gerçeker AA. A In-vitro activities of various antibiotics, alone and in combination with amikacin against Pseudomonas aeruginosa. Int J Antimicrob Agents. 2001;18(6):567-570.

4. Govan JR, Deretic V. Microbial pathogenesis in cystic fibrosis: mucoid Pseudomonas aeruginosa and Burkholderia cepacia. Microbiol Rev. 1996;60(3):539-574.

5. Silby MW, Winstanley C, Godfrey SA, et al. Pseudomonas genomes: diverse and adaptable. FEMS Microbiol Rev. 2011;35(4):652-680.

6. Gordillo F, Chávez FP, Jerez CA. Motility and chemotaxis of Pseudomonas sp. B4 towards polychlorobiphenylsand chlorobenzoates. FEMS Microbiol Ecol. 2007;60(2):322-328.
7. O'Toole GA, Kolter R. Initiation of biofilm formation in Pseudomonas fluorescens WCS365 proceeds via multiple, convergent signal-ling pathways: a genetic analysis. Mol Microbiol. 1998;28(3):449-461.

8. Costerton JW, Stewart PS, Greenberg EP. Bacterial biofilms: a common cause of persistent infections. Science. 1999;284(5418):1318-1322.

9. Vater SM, Weiße S, Maleschlijski S, et al. Swimming Behavior of Pseudomonas aeruginosa Studied by Holographic 3D Tracking. PLoS One. 2014;9(1):e87765

10. Köhler T, Curty LK, Barja F, et al. Swarming of Pseudomonas aeruginosa is dependent on cell-to-cell signaling and requires flagella and pili. J Bacteriol. 2000;182(21):5990-5996.

11. Van Delden C, Iglewski BH. Cell-to-cell signaling and Pseudomonas aeruginosa infections. Emerg Infect Dis. 1998;4(4):551-560.

12. Gilbert P, Das J, Foley I. Biofilm susceptibility to antimicrobials. Adv Dent Res. 1997;11(1):160-167.

13. Stewart PS. Mechanisms of antibiotic resistance in bacterial biofilms. Int J Med Microbiol. 2002;292(2):107-113.

14. Feldman M, Bryan R, Rajan S, et al. Role of flagella in pathogenesis of Pseudomonas aeruginosa pulmonary infection. Infect Immun. 1998;66(1):43-51.

15. Rashid MH, Kornberg A. Inorganic polyphosphate is needed for swimming, swarming and twitching motilities of Pseudomonas aeruginosa. Proc Natl Acad Sci U S A. 2000;97(9):4885-4890.

16. Déziel E, Lépine F, Milot $\mathrm{S}$, et al. rhlA is required for the production of a novel biosurfactant promoting swarming motility in Pseudomonas aeruginosa: 3-(3-hydroxyalkanoyloxy) alkanoic acids (HAAs), the precursors of rhamnolipids. Microbiology. 2003;149(Pt 8):2005-2013.

17. Lyte M, Freestone PP, Neal CP, et al. Stimulation of Staphylococcus epidermidis growth and biofilm formation by catecholamine inotropes. Lancet. 2003;361(9352):130-135.

18. Freestone P. Communication between bacteria and their hosts. Hindawi Publishing Corporation Scientifica. 2013;2013:15.

19. Freestone PP, Hirst RA, Sandrini SM, et al. Pseudomonas aeruginosa-catecholamine inotrope interactions: a contributory factor in the development of Ventilator Associated Pneumonia? Chest. 2012;142(5):1200-1210

20. Singh PK, Schaefer AL, Parsek MR, et al. Quorum-sensing signals indicate that cystic fibrosis lungs are infected with bacterial biofilms. $\mathrm{Na}$ ture. 2000;407(6805):762-764.

21. Jimenez PN, Koch G, Thompson JA, et al. The multiple signaling systems regulating virulence in Pseudomonas aeruginosa. Microbiol Mol Biol Rev. 2012;76(1):46-65. 